le portiQue Le Portique

Revue de philosophie et de sciences humaines

$30 \mid 2013$

Écrire sur l'art

\title{
Georges Dumézil, un parcours en mots
}

Georges Dumézil, a journey in words

Der mit Wörtern ausgedrückte Lauf des Lebens und der Forschung

\section{Jean-François Bert}

\section{OpenEdition}

\section{Journals}

Édition électronique

URL : http://journals.openedition.org/leportique/2649

DOI : $10.4000 /$ leportique.2649

ISSN : $1777-5280$

Éditeur

Association "Les Amis du Portique"

Édition imprimée

Date de publication : 18 juillet 2013

ISSN : 1283-8594

\section{Référence électronique}

Jean-François Bert, « Georges Dumézil, un parcours en mots », Le Portique [En ligne], 30 | 2013,

document 1, mis en ligne le 01 juillet 2015, consulté le 26 mars 2021. URL : http://

journals.openedition.org/leportique/2649; DOI : https://doi.org/10.4000/leportique.2649

Ce document a été généré automatiquement le 26 mars 2021.

Tous droits réservés 


\title{
Georges Dumézil, un parcours en mots
}

\author{
Georges Dumézil, a journey in words \\ Der mit Wörtern ausgedrückte Lauf des Lebens und der Forschung
}

Jean-François Bert

1 Deux ans avant sa mort, en 1984, Georges Dumézil donna deux entretiens filmés dans son appartement de la rue Notre-Dame-des-Champs pour l'émission « Rencontres » de Radio Canada ${ }^{1}$. Qu'est-ce que ces deux émissions de trente minutes ${ }^{2}$ chacune nous apprennent de nouveau sur cet auteur devenu aujourd'hui un «classique » des sciences humaines et sociales pour avoir profondément renouvelé les méthodes d'investigation historique et, plus généralement, la connaissance du fonctionnement de l'esprit humain?

2 Étonnamment, ces deux entretiens ne font pas apparaître une facette oubliée ou inconnue de l'érudit polyglotte qui, depuis ses premiers travaux sur l'ambroisie, le crime des Lemniennes ou le problème des centaures ${ }^{3}$, s'est dit animé par le sentiment de l'existence d'une concordance entre la triade précapitoline des anciens romains et la division de la société indienne arya entre les brâhmana, les râjanya ou ksatriyaet les Vaiçya. Une concordance qui lui est apparue clairement en 1938 après avoir pris connaissance des travaux de Kretschner et de Vendryes sur le vocabulaire religieux indo-iranien et italo-celtique.

3 Rien, non plus, n'est dit sur l'intense débat qui eut lieu après la dénonciation politique de Dumézil, au début des années 1980, soulevée par le couple d'historien MomiglianoGinzburg et dont le résultat a été de durablement déplacer les enjeux scientifiques de ses analyses ${ }^{4}$.

4 Pourtant, il faut considérer les nombreuses " prises » de parole que Dumézil fit, entre 1984 et 1986, comme révélatrices d'une partie de son parcours et surtout de son travail. Devant Didier Éribon, François Ewald, Bernard Pivot, mais aussi Jean-Pol Hocq pour la RTBF et Marcel Brisebois pour Radio Canada ${ }^{5}$ qui ont chacun interrogé l'« ultrahistorien" au soir de son existence, celui-ci raconta le fil d'une vie qui, comme 
beaucoup d'autres, a été faite de détours, d'occultations et d'épiphanies. Une vie de «savant» qui a été tout entière tournée vers le travail. Vieetœuvres'entremêlantdefaçoninextricable.

5 Aussi, l'œuvre et le parcours de Dumézil mériteraient d'être relus à partir des paroles dites qui nous rendent palpable le dynamisme de ses recherches; entendu qu'une « recherche» recouvre, en plus des travaux publiés, l'ensemble des efforts entrepris pour acquérir, obtenir quelque chose ou s'en approcher. Chaque nouveau livre, chaque nouvel article, chaque nouvel entretien lui a donné l'occasion de proposer de nombreuses corrections, quelques détournements et ajouts, mais aussi - chose plus rare - des repentirs. Ce rapport tout à fait singulier de Dumézil à sa propre écriture, à son propre travail, s'explique à la fois par la nature mouvante de son objet - puisqu'à chaque nouvelle découverte concernant les Indo-Européens, il a été obligé de modifier le sens des structures qu'il avait cherché à décrire -, et par sa manière de travailler l'histoire de nos origines les plus profondes. En effet, seule l'accumulation lente et progressive des faits lui a permis d'avérer, de corriger ou de rejeter ${ }^{6}$ les fragments de l'héritage indo-européen qu'il pouvait déceler derrière les mauvais tours de l'histoire jusque dans nos faits divers les plus contemporains.

\section{De la vie et de l'œuvre scientifique}

6 Ce rapport tout à fait particulier que Dumézil entretint avec son œuvre est d'autant plus important que l'écriture scientifique laisse encore trop peu de place à la complexité d'une démarche, aux détails d'une description et surtout aux «tâtonnements $~{ }^{7}-$ terme par lequel Dumézil se plaisait à définir sa manière de faire. L'écriture scientifique n'est pas faite non plus pour rendre compte de la finesse et de la fragilité d'une définition, d'une hypothèse, et peut-être d'abord, concernant l'historien, de la volonté - grâce à une comparaison génétique - d'établir des rapprochements, de faire surgir des homologies, de retrouver des parentés formelles dans des champs de savoir pourtant très différents. Comme il le précisa à Mircea Eliade qui était lui aussi animé par ce goût pour les précisions et l'utilité de la démarche comparative: «je compare des choses génétiquement apparentées qui dérivent qu'un même prototype et qui sont observables sur un domaine limité [...] ; j'essaie de déterminer l'évolution qui a mené de ce prototype aux divers états historiquement attestés " 8 .

7 Depuis 1938, Dumézil n'a cessé de lister les risques d'une telle méthode, dessinant les contours de son usage, mais rappelant aussi qu'elle ne doit pas interdire une certaine dose d'imprudence dans la recherche, imprudence que le savant doit savoir corriger par de l'humilité. C'est ce qu'il précise, par exemple, dans l'introduction de son Mythe et épopée I, en rappelant que son travail avait été, depuis les années Trente, de multiplier : « les retouches, les rétractations, les confirmations, et aussi les défenses et les contreattaques, gardant le sentiment bien plaisant que la matière était entre mes mains indéfiniment malléable et perfectible ${ }^{9}$. Dans l'entretien de 1984 pour Radio Canada, il est encore plus clair sur ce point : "Votre ceuvre est colossale, elle suscite l'admiration de tous. Elle a aussi provoqué des réactions, des oppositions. Comment réagissez-vous à ses oppositions? :Très différemment. Si elles sont méchantes, je réagis violemment. Si elles sont justes, en général, je commence par leur résister et ensuite je leur cède. Mon œuvre est pleine de repentir. Ce n'est pas forcément un repentir par tête-à-queue, mais c'est une retouche, c'est un complément, c'est ici quelque chose qu'il faut effacer, là 
qu'il faut ajouter... Évidemment, j'ai une propension à faire surtout des améliorations. D'autres, à faire des corrections. Mais nous sommes tous des infirmes. Je fais mon œuvre et puis l'avenir dira. Les gens qui viennent derrière moi nettoieront ».

\section{Le motif biographique}

8 À première vue anecdotique et insignifiant, le récit biographique a été pour tous les derniers interviewers de Dumézil un instrument privilégié pour introduire et expliquer son parcours sinueux, pour expliquer lesrapports souvent complexes entrelavieetl'œuvre : de son poste de lecteur à l'université de Varsovie en 1921, à son élection au collège de France en 1948, en passant par son départ pour la Turquie en 1925, puis pour la Suède en 1931, ou encore l'aventure de son élection à l'École pratique des hautes études en 1933. C'était sans compter sur le fait que Dumézil maitrisait l'art de réévaluer sa vie sans être dupe de la valeur de l'exercice convenu du "grand» entretien qui permet de révéler autant que de dissimuler. Un jeu d'autant plus facile qu'il s'agissait, avec Dumézil, d'une vie qui a été profondément liée à la fréquentation des livres. C'est en classe de $5^{e}$, après avoir lu Horace et les Curiace qu'il se dit frappé par l'existence d'une analogie entre Horace tuant trois adversaires et Hercule tuant un monstre à trois têtes. La lecture d'Hercule et Cacus, publiéen 1863 par Bréal, devient lui aussi un de ses livres modèles comme, en 1913, le livre de Perrin sur les atomes. Dumézil, on l'oublie trop souvent, était homme de science : «aujourd'hui, je suis très attentif à ce qui se passe en biologie ou en physique. Elle est là, la philosophie ! Dans les livres de François Jacob, Jean-Pierre Changeux, Jacques Ruffié... Vous savez, si avant de dormir, je prends du Ruffié, je tiens jusqu'à minuit, si je prends un roman, je tombe à onze heures ${ }^{10}$. Plus tard, ce sera la découverte des épopées populaires russes durant sa mobilisation en 1917 qui lui donnera la matière première pour son sujet de thèse. Son style d'écrivain n'est pas exempt de références précises. L'Essai sur les mœurs de Voltaire lui a appris "à faire court », Chateaubriand lui a servi, au contraire, «à faire long ». Enfin, et pour clore ce point, c'est l'absence de bibliothèques suffisamment fournies sur les Indo-Européens qui, durant son séjour à Istanbul, l'a poussé à étudier les Ossètes, derniers survivants des Scythes et des Sarmates de l'Antiquité. C'est en véritable botaniste qu'il se mit alors à sauver les langues Oubykh, Beslemey et Laze ${ }^{11}$.

\section{Unifier un parcours}

Dumézil excellait dans un autre exercice contraint des vies savantes, ces vies traverséesd'unetensionentrelavieetl'œuvre,l'uneetl'autres'entremêlantdefaçoninextricable ${ }^{12}$. En 1967, un an avant sa retraite du Collège de France, il donnera sa propre version de son parcours, relisant et reliant chacune des périodes de sa vie : "Jusqu'en 1938, j'ai cherché sans trouver et en refaisant pour mon propre compte les erreurs de l'école de Max Müller, d'Adalbert Kuhn ou de Victor Henry : j'ai imprimé pas mal de bêtises, que je suis obligé de rejeter quant au fond. Puis à partir de 1938 s'est ouverte une période nouvelle marquée par la "découverte" du système de la tripartition, comme structure déterminante $\mathrm{du}$ système de représentation indo-européen, dont j'espère que les acquisitions resteront plus valables. Et maintenant, eh bien, je donne mon "testament" : j'arrive à l'âge où il faut donner une forme définitive à certaines idées » ${ }^{13}$. Dumézil n'a eu de cesse de répéter dans ses entretiens radiophoniques ou télévisés qu'il 
n'était pas un homme engagé ni un homme d'action et qu'il n'a jamais cherché à faire école. Son travail ne servirait finalement à rien: "je suis irrémédiablement individualiste ", affirme-t-il à François Ewald ${ }^{14}$. Il est encore plus impertinent devant un Pivot médusé par sa réponse: « B.P. - À quoi ça sert ? G.D. - À rien. B.P. - Comment, ça sert à rien? G.D. - D'abord je professe que les sciences sociales ne sont pas mûres ; quand on saura comment fonctionne le cerveau, on pourra savoir comment les gens évoluent, mais jusqu'à ce qu'on le sache... ${ }^{15}$. Il lèvera finalement toute ambiguïté concernant le rôle qu'il convient de donner à ses découvertes devant Éribon : "à supposer que j'aie totalement tort, mes Indo-Européens seront comme les géométries de Riemann et de Lobatchevsky: des constructions hors du réel. Ce n'est déjà pas si mal. Il suffira de me changer de rayon dans les bibliothèques: je passerai dans la rubrique roman ${ }^{16}$.

Cette position hors du temps, dégagée pour ainsi dire, n'est pas qu'ironie ou fausse modestie. C'est grâce à elle qu'il a pu reprendre sans relâche ses hypothèses et ses analyses, rappelant à maintes reprises les erreurs de ses premiers ouvrages qu'il écrit avec en tête l'idée « simpliste » que le social est la clef d'explication de l'idéologie. Une position qu'il change définitivement en 1950 en démontrant que le déterminisme n'explique rien, ni les représentations, ni la société. Il ne permet pas plus de comprendre la conservation d'une idéologie dans une société dans laquelle l'idéologie ne se réalise pas. La tripartition fonctionnelle n'est pas le reflet d'une structure économique ou sociale et ne s'accompagne pas forcément, comme en Inde, d'une division réelle de la société. Décrochée de sa réalisation, la tripartition peut tout aussi bien être réelle qu'idéale. Le cas de la déesse Aurore est sur ce point particulièrement exemplaire et Dumézil se fera fort de le mettre en scène à plusieurs reprises: les femmes romaines qui, le 11 juin, font entrer une femme esclave pour la battre avec des verges dans le temple de Mater Matuta miment un mythe de Usas, l'aurore védique. Ce qui est rituel à Rome est relaté mythiquement en Inde.

\section{Répondre aux critiques}

11 Il ne faut cependant pas s'y tromper; les autocritiques que Dumézil fait lors des entretiens ne sont pas la marque d'une incohérence ou, pis encore, d'une instabilité d'humeur de son auteur. Elles sont d'abord des réponses données aux nombreuses critiques que Dumézil groupe selon deux catégories distinctes ${ }^{17}$. Tout d'abord, celles qui s'attaquent de front à sa rhétorique et qui soulignent qu'un grand nombre de ses triades ne sont trifonctionnelles que par des artifices d'interprétation. Les similitudes que Dumézil remarque ne seraient que des coïncidences ou au mieux le résultat d'une évolution parallèle. De toute façon, précisent les plus sceptiques, aucune observation directe des Indo-Européens n'est plus possible, aucune vérification ne peut plus être sérieusement envisagée. Il s'en défendra comme dans l'introduction de son dernier Mythe et épopée. Parlant de la "théorie dumézilienne ", il rappelle qu'elle "consiste en tout et pour tout à rappeler qu'il a existé, à un certain moment, des Indo-Européens et à penser, dans le sillage des linguistes, que la comparaison des plus vieilles traditions des peuples qui sont au moins partiellement leurs héritiers doit permettre d'entrevoir les grandes lignes de leur idéologie. À partir de là, tout est observation $[. ..]{ }^{18}$. Certes, la structure tripartite a cessé de fonctionner et il n'existe pas de "résurgences", de «survivances » ou de "fantômes" de cette grande idéologie commune aux peuples 
Indo-Européens disparus, cependant elle n'est pas une coquille vide. L'idéologie peut continuer de recevoir des contenus différents. Elle peut se renouveler en s'immergeant dans un nouveau milieu.

Les autres « attaques » s'accordent pour refuser de croire que les triades mises en avant par Dumézil sont la preuve d'un passé commun. La structure tripartite est dans la nature même des choses. Dumézil ne cherche pas à remettre en cause l'argument. Lui aussi pense que les trois fonctions existent dans tous les organismes. Il précise cependant que peu de sociétés ont réellement pris conscience de l'existence d'une telle structure naturelle au point d'en faire une idéologie qui justifie les conduites, oriente la pensée, la littérature, le rituel, et, d'une façon plus générale, la religion. Seuls les peuples Indo-Européens ont eut conscience de cette conception des rapports humains ou divins fondée sur la distinction entre une souveraineté magique et juridique, la force et la puissance guerrières, et la fécondité et la fertilité (végétales, animales et humaines). Certains de ces peuples ont même réalisé cette distinction, certes les Indiens et les Iraniens avant la réforme de Zoroastre, mais aussi les Celtes. En revanche, rappelle Dumézil, ni l'Égypte, ni Sumer, ni les Hébreux, ni les Chinois, ni les Turcs, ni les Sibériens, ni les Finno-Ougriens n'ont construit de telles conceptions du monde et donc n'ont exprimé cette structure dans leurs images, leurs récits, leurs épopées ou leur symbolisme... Dans un entretien donné en 1978, il précise sur ce point que la nécessité de commandement, de la protection physique et de l'abondance ou de la production en tous domaines sont effectivement des besoins universels, "mais ressentir des besoins est une chose, réfléchir sur eux pour en faire une structure intellectuelle et un moule de pensée en est une autre. Or ce qui fait l'originalité des Indo-Européens, c'est qu'ils en ont fait l'épine dorsale philosophique et "idéologique » de toute leur conception du monde $"{ }^{19}$.

13 La réponse parait simple, imparable. Elle n'emmènera pourtant pas l'adhésion de tous ceux pour qui Dumézil reste bien plus proche d'un affabulateur que d'un historien qui décide, comme il le dit, de s'occuper de faits idéologiques qui possèdent leur propre temporalité et leur indépendance vis-à-vis de l'histoire événementielle. Une histoire, précise-t-il encore, qui plus que toutes les autres doit se faire « sans idée préconçues au départ » et « sans espérance, à l'arrivée, de résultats universellement valables » ${ }^{20}$.

\section{NOTES}

1. Présenté par Marcel Brisebois, l'émission Rencontres a été diffusée sur radio Canada entre 1971 et 1989. La première émission avec Dumézil aborde la question de son activité de mythologue, sur le contexte intellectuel de ses premiers travaux et sur la portée de sa méthode comparative. La seconde revient surtout sur l'hypothèse centrale de toute l'œuvre de l'historien des religions : la tripartition fonctionnelle et la réception de ses travaux qui ont souvent suscité de l'hostilité et de l'incompréhension.

2. Ces deux émissions peuvent être visionnées sur plusieurs sites, dont http://www.scoop.it/t/ archivance/p/3288643557/georges-Dumézil-par-lui-meme-1-2 (dernière visite : le 12 décembre 2012), ou encore celui de Marcel Brisebois, http://www.dieu-parmi-nous.com/remission.html. 
3. Le Festin d'immortalité. Étude de mythologie comparée indo-européenne, Paris, Geuther, Annales du Musée Guimet, 1924 ; Le Crime des Lemniennes. Rites et Légendes du monde égéen, Paris, Geuther, Annales du Musée Guimet, 1924 ; Le Problème des Centaures. Étude de mythologie comparée indoeuropéenne, Paris, Geuther, Annales du Musée Guimet, 1929.

4. Sur ce point, indiquons seulement que le fait que Dumézil n'utilisa jamais la notion de «mentalité » ou même le terme d' "Aryen » pour nommer les peuples indo-européens aurait dû suffire à empêcher de situer son œuvre en fonction d'opinions politiques ou religieuses. Voir Carlo GINZBURG, « Mythologie germanique et nazisme. Sur un livre ancien de Georges Dumézil », Annales, Année 1985, p. 695-715.

5. "Le messager des dieux », entretien avec François Ewald, Le Magazine littéraire, avril, 1986, p. 16-21; Apostrophes, numéro spécial consacré à Georges Dumézil, 18 aout 1985; "Georges Dumézil par lui-même ", Inédits de la RTBF...

6. Notons cette réponse de Dumézil faite lors de cette émission de Radio Canada : « Vous comparez des langues, vous comparez du vocabulaire, vous comparez des mots... C'est ça que le XIX ${ }^{\mathrm{e}}$ siècle a fait. Mais dés le début des gens se sont dit : les peuples qui parlent des langues si proches ne peuvent pas ne pas avoir en commun autre chose que la langue, c'est-à-dire de la pensée. De la pensée exprimée dans de la littérature, dans de la religion, dans des institutions... J'ai repris avec quelques autres - je ne suis pas seul du tout - au début de ce siècle, vers 1920, la question mais avec les armes que l'ethnographie et les progrès des sciences sociales fournissaient. Et, effectivement, après beaucoup de tâtonnements, nous sommes tombés sur des structures de pensée communes à sinon tout l'ensemble de la famille, du moins à des parties très considérables de l'ensemble".

7. Notons cette réponse de Dumézil faite lors de cette émission de Radio Canada : « Vous comparez des langues, vous comparez du vocabulaire, vous comparez des mots... C'est ça que le XIX siècle a fait. Mais dés le début des gens se sont dit: les peuples qui parlent des langues si proche ne peuvent pas ne pas avoir en commun autre chose que la langue, c'est-à-dire de la pensée. De la pensée exprimée dans de la littérature, dans de la religion, dans des institutions... J'ai repris avec quelques autres - je ne suis pas seul du tout - au début de ce siècle, vers 1920, la question mais avec les armes que l'ethnographie et les progrès des sciences sociales fournissaient. Et, effectivement, après beaucoup de tâtonnements, nous sommes tombés sur des structures de pensée commune à sinon tout l'ensemble de la famille, du moins à des parties très considérables de l'ensemble».

8. G. DUMÉZIL, "Ces religions dont nous héritons ", dialogue avec Mircea Eliade, Les Nouvelles littéraires, 25 octobre 1978, p. 18.

9. DUMÉZIL, Mythe et épopée, I, p. 48 (édition quarto).

10. «Accepter d'être du terreau pour le jardin des autres », entretien avec Jean-Pierre Salgas, $L a$ Quinzaine littéraire, 16-31 mars 1986.

11. Le verbe Oubykh, études descriptives et comparatives, avec la collaboration de Tevfik Esenç, Mémoires de l'Académie des Inscriptions et Belles Lettres, Paris, 1975.

12. Dans l'entretien de 1984, Brisebois aborde ce point: "Je vais vous poser une question qui peut apparaître cruelle mais que, nécessairement, le profane se pose. Pourquoi faire une cuvre pareille? Pourquoi avez-vous mis toute votre vie dans cette ceuvre? Demandez à un peintre pourquoi il se met sur son chevalet. Il y a certainement une affaire ou de prédisposition, ou d'habitude? J'ai toujours beaucoup aimé les mythes. Quand j'étais gamin, je vivais aussi bien avec l'histoire d'Hercule qu'avec des comtes de fées. Ça m'a toujours intéressé. Si mon orientation avait été différente, j'aurais peut-être été poète. J'aurai écrit des choses, enfin si j'en avais eu le talent, comme les strophes de l'Énéide qui mettent en en scène Hercule, l'amour grandissant avec le crépuscule... ».

13. «Entretien avec Georges Dumézil », La Religion archaïque des Romains, propos recueillis par J.P. Gorin, Le Monde, 15 mars 1967. 
14. Op. cit., p. 20.

15. Op. cit, $46,16 \mathrm{~min}$.

16. Georges DUMÉzIL, Entretiens avec Didier Éribon, 1987, Paris, Folio, p. 220.

17. Dumézil aborde pour Radio Canada la question difficile de la continuation de son travail : « Il $y$ a deux espèces de gens que mon travail intéresse. Des gens que j'aimerais mieux ne pas voir s'en occuper parce qu'ils disent n'importe quoi. Il y a des gens qui s'imaginent que tout cela est très facile et qu'il suffit de baisser la main pour ramasser les trois fonctions. Non. Il faut faire un minimum d'autocritique que la plupart ne font pas. Aussi bien en France qu'en Amérique. »

18. DUMÉzIL, Mythe et épopée, III, p. 1085 (quarto).

19. «Georges Dumézil : l'explorateur de nos origines », entretien recueilli par Alain de Benoist, présentation de Jean Varenne, Le Figaro-dimanche, 29-30 avril 1978.

20. Georges DumÉzIL, Mythe et épopée, III, p. 14.

\section{RÉSUMÉS}

L'histoire récente des sciences humaines et sociales commence à prendre en considération la question des représentations de la vie savante par les savants eux mêmes, comme de la manière dont il parle et commente leur propre travail de recherche. Georges Dumézil donne à voir un parcours très intéressant, faits de multiples repentirs et tâtonnements que Dumézil intégra au cœur de sa méthode comparative.

The recent history of humanities and social sciences is starting to take into account the question of the representations of a life of science made by scientists themselves. George Dumézil in this respect presents a fascinating journey, made of multiple pentimentos, regrets and fumblings the scientist absorbed into his comparative method.

Die Geisteswischenschaften nehmen jetzt Rücksicht auf die Frage der Vorstellungen in Betreff des gelehrten Lebens und der Forschung, welche die Gelehrten selbst berücksichtigen. Georges Dumézil lehrt uns, dass seiner Arbeit Leben zu seiner Methode gehört.

\section{AUTEUR}

\section{JEAN-FRANÇOIS BERT}

Jean-François Bert est Maître d'enseignement et de recherche à l'Université de Lausanne. Membre de l'IRCM (Institut religions, cultures, modernité) et chercheur associé au Labex HASTEC (Histoire et anthropologie des savoirs, des techniques et des croyances), ses principaux travaux portent sur l'histoire des pratiques savantes. Dernière publication : L'Atelier de Marcel Mauss, Paris, Éditions du CNRS, 2012. 